\title{
Racing Thoughts
}

National Cancer Institute

\section{Source}

National Cancer Institute. Racing Thoughts. NCI Thesaurus. Code C117265.

Abnormally rapid thoughts with or without abrupt changes from one topic to another. 